\title{
Un estudio prospectivo define variables útiles para estratificar el riesgo embólico en pacientes con estenosis mitral
}

\author{
Predictors of systemic embolism in patients with mitral stenosis. Chiang CW, Lo SK, Ko YS et al. Ann. Intern. Med. $1998,128: 885-89$.
}

\begin{abstract}
Objetivo
Identificar prospectivamente variables asociadas al desarrollo de fenómenos embólicos en pacientes con estenosis mitral.
\end{abstract}

\section{Diseño}

Estudio prospectivo de cohortes.

\section{Lugar}

Instituciones médicas asociadas a Universidades de EE.UU.

\section{Pacientes}

534 pacientes consecutivos de edad mayor a 15 años con área valvular mitral en el ecocardiograma bidimensional menor o igual a 2,0 cm2 que consultaron entre abril de 1987 a diciembre de 1994 .

132 se encontraban en ritmo sinusal y 402 presentaban fibrilación auricular. Se excluyeron pacientes con endocarditis infecciosa y aquellos con estado clínico crítico que motivó su fallecimiento.

\section{Evaluación de factores pronósticos}

Fueron evaluadas nueve variables clínicas (edad, sexo, embolia previa,

fibrilación auricular, hipertensión arterial, insuficiencia cardíaca Clase Funcional III-IV, tratamiento anticoagulante, historia de comisurotomía mitral percutánea o cirugía valvular) y diez ecocardiográficas (área mitral, diámetro de fin de sístole, trombo en aurícula izquierda, insuficiencia aórtica, deterioro de la función sistólica del ventrículo izquierdo, etc.) para estimar el riesgo embólico durante el seguimiento.

\section{Medición de resultados principales}

El punto final del estudio fue la ocurrencia de un nuevo fenómeno embólico durante el seguimiento. El diagnóstico del mismo se basó en el desarrollo de síntomas y signos de isquemia arterial periférica de instalación súbita o manifestaciones neurológicas sin pródromos; o en los

Fuente de financiamiento:

\section{COMENTARIO}

El presente trabajo constituye un importante soporte de las recomendaciones para la prevención de fenómenos embólicos en pacientes con estenosis mitral recientemente publicadas por el comité de expertos en valvulopatías del American College of Cardiology / American Heart Association Task Force.

Se objetiva en este trabajo prospectivo que las variables de mayor importancia para estratificar el riesgo embólico son la edad, la presencia de fibrilación auricular y el antecedente de embolismo previo. El hallazgo del rol "protector" de la comisurotomía mitral percutánea, concuerda con las observaciones de trabajos retrospectivos, en los que se objetivaba reducción del estasis sanguíneo y del estado de hipercoagulabilidad secundaria local a nivel de la aurícula izquierda luego de la realización de este procedimiento.

La objetivación del mayor riesgo embólico en pacientes en ritmo sinusal que tienen trombo visible en la aurícula izquierda y/o insuficiencia aórtica, constituye un hallazgo de relevancia. Sin embargo, el comité de expertos (ACC/AHA) no consideró a la presencia de in-

*Ver Glosario

\section{Dr. Ernesto Ferreirós}

Servicio de Cardiología. Hospital Italiano de Buenos Aires. hallazgos de la tomografía computada, angiografía cerebral o cirugía.

\section{Resultados principales}

El seguimiento fue de $36,9 \pm 22,5$ meses. De los 534 pacientes, 257 $(48,1 \%)$ recibieron tratamiento anticoagulante durante el período de seguimiento. 60 pacientes $(11,2 \%)$ presentaron embolismo sistémico en el seguimiento. El análisis multivariable* demostró una correlación significativa entre la presencia de fibrilación auricular y el riesgo embólico. Como el efecto pronóstico de cada variable era distinto en presencia-0 ausencia de fibrilación auricular se analizaron separadamente los subgrupos de pacientes en ritmo sinusal o con fibrilación auricular (ver Tabla).

\begin{tabular}{lll}
\hline Variable & RR (IC 95\%) & p \\
\hline Pacientes en ritmo sinusal $(n=132)$ & & \\
\hline Edad (por década) & $1,12(1,04-1,21)$ & 0,002 \\
\hline Area valvular mitral $\left(<1,0 \mathrm{~cm}^{2}\right)$ & $16,9(1,53-187,0)$ & 0,021 \\
\hline Trombo en aurícula izquierda & $37,1(2,82-487,8)$ & 0,006 \\
\hline Insuficiencia Aórtica & $22,4(2,72-184,8)$ & 0,004 \\
\hline Pacientes con fibrilación auricular $(n=402)$ & & \\
\hline Comisurotomía mitral percutánea & $0,37(0,18-0,79)$ & 0,01 \\
\hline Embolia previa & $3,11(1,66-5,85)$ & $<0,001$ \\
\hline
\end{tabular}

Conclusiones

La indicación de anticoagulantes orales en pacientes con estenosis mitral en presencia de fibrilación auricular o embolismo previo queda plenamente justificada por los hallazgos del presente estudio.

Según los resultados, la indicación debería hacerse extensiva a pacientes con trombo visible en aurícula izquierda o insuficiencia aórtica significativa. La comisurotomía mitral parece conferir protección para el desarrollo de fenómenos embólicos en esta población.

\section{Referencias}

1. Guidelines for the management of patients with valvular heart disease: Executive summary. A report of the American College of Cardiology / American Heart Association Task Force. Circulation 1998, 98: 1949-1984

2. Benjamin E.J., Levy D., Vaziri S.M., y col. Independent risk factors for atrial fibrillation in a population based cohort. The Framingham Heart Study. J. A. M. A. 1994, 271: 840-844

3. Hwang J.J., Kuan P., Lin S.C., y col. Reappraisal by transesophageal echocardiography of the significance of left atrial thrombi in the prediction of systemic arterial embolization in rheumatic mitral valve disease. Am. J. Cardiol. 1992, 70: 769-773

4. Peverill R.E., Harper R.W., Harris G., y col. Amelioration of regional left atrial hypercoagulability by balloon mitral valvuloplasty in patients with mitral stenosis. Circulation 1995,92 (suppl. 1): 1-21 